\title{
Investigations on testing and topologies of grid connected PV Inverters- A Comprehensive Review
}

\author{
Bandla Pavan Babu ${ }^{1}$, Ashok Kumar L ${ }^{2}$, V. Indragandhi ${ }^{3}$ \\ \{ pavanbabu89@gmail.com ${ }^{1}$, lak.eee@psgtech.ac.in ${ }^{2}$, indragandhi.v@vit.ac.in $\left.{ }^{3}\right\}$ \\ School of ElectricalEngineering,VIT,Vellore ${ }^{1,3}$, PSG College of Technology, Coimbatore, India ${ }^{2}$
}

\begin{abstract}
The main cause of the Electrical power conversion which is generated with the help of renewable energy (Solar) is the Photo Voltaic (PV) inverter. This PV inverter performs a vital role in conversion of the electrical power in to the required i.e., from DC to AC by maintaining the standards which is synchronized to Grid or directly to the appliances. Designing of the PV inverter should be considered with the performance, safety and interconnection of grid characteristics of PV systems. Otherwise this may reflect on the entire system making it to an abnormal and also becomes a cause in increase of the severity. IEEE 1547 standard for the integration of energy resources with electrical power systems is considered. In this paper, a complete review on the test instructions, islanding and power quality which are to be considered in PV inverter as per the standards are presented. This review work also gives complete information of PV inverter performance at different levels with a clear step by procedure which provides a complete information in maintain the standard tests and helpful of further developments.
\end{abstract}

Keywords: Renewable Energy Sources; PV Inverter and Power Quality.

\section{Introduction}

The growth of renewable power generation due to increase in power demand has grown exponentially from the past few years [1-3]. Therefore more amount of solar power generation is allowed which are coordinated with the grid effectively for meeting the power demand. The Electric grid response in coordination with solar generation is a function of dynamic responses. Due to this massive incorporation of the solar power generation, there is a need for proper study on the PV inverter which is a major device in power conversion [4-7]. Figure 1 represents the setup of PV inverter for conducting the various tests.

This paper focuses on the step by step procedure of the various test instructions, islanding and power quality which are to be considered in PV inverter as per the standards. In section 2 Testing Process, In Section 3, Inverter Output Time Delay Test, Anti-Island test of Grid-tied Power Converters, Voltage Tests, Frequency Tests and Harmonic test are explained. In section 4, Inverter DC Performance Test, in Section 5, Performance Tests of Inverter (AC), in Section 6, 3-phase inverter ac performance tests, in section 7 Challenges and Issues in grid connected PV inverters, in Section 8, Configurations of PV Inverter in section 9, Different topologies used in Grid tied PV Inverters and in section 10 are explained. 


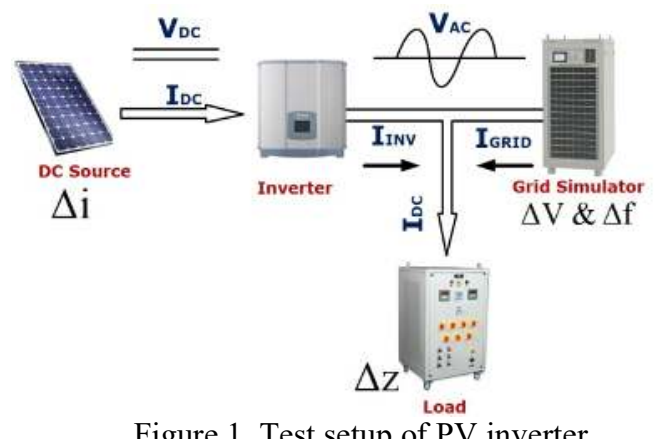

Figure 1. Test setup of PV inverter

\section{Testing Process}

The objective of this work is to analyse the impact of power quality perturbations on various power converter based sources such as, PV system, micro-grid, energy storage system, and V2G system as shown in Figure 2. Each of these source integrate with voltage source converter modules, control boards, sensors and protection circuits. Employing the regenerative grid emulator, the power quality perturbations like, voltage transients, voltage and frequency variation, voltage and frequency fluctuations, harmonics, and unbalances are emulated [8-12]. During the injection of power quality perturbations, the behavioural analysis and life span estimation of switches and converter modules have to be carried out using specific search algorithm. All systems are linked to the regenerative grid emulator via a power hub switch that connects single or multiple sources simultaneously. FPGA based controller controls the switching order of sources, also through sensors and data acquisition system, it records the electrical quantities for analysing the behaviour and life span of the converter. Additionally, high accuracy power quality analysers are connected to individual sources for online analysis. The behavioral performance of the converter has to be analysed by conducting the tests discussed from section III.

\section{Test Plan}

\section{A. Time Delay Test of Inverter Output}

The test is performed for determining the ramp-up and ramp-down rate time of an inverter [13]. The time taken to start generating output when a DC source is connected is the ramp-up time. Similarly, the time taken to reach the minimum current output when the inverter is isolated from DC source is the ramp-down time. Any other sources like PV solar and battery can be the de input of inverter.

i. Determining ramp up and down time constant during start up and shutdown

The purpose of this investigation is to identify ramp up and down time constant of an inverter during sudden disconnection and connection in the grid. Particularly, in the course of power generation, an immediate start-up or shut down of input DC source. The inverter test has to be performed in the following sequences; 
- Initially set the steady-state voltage and frequency in regenerative grid emulator and then connect the inverter to it.

- Attach the DC supply to the inverter input and write down the amount of time taken to achieve its nominal source or output current.

- Switch the inverter and take note of the time taken to reach the required output current.

- Repeat the test multiple times to ensure the ramp-down and ramp-up delay time is consistent.

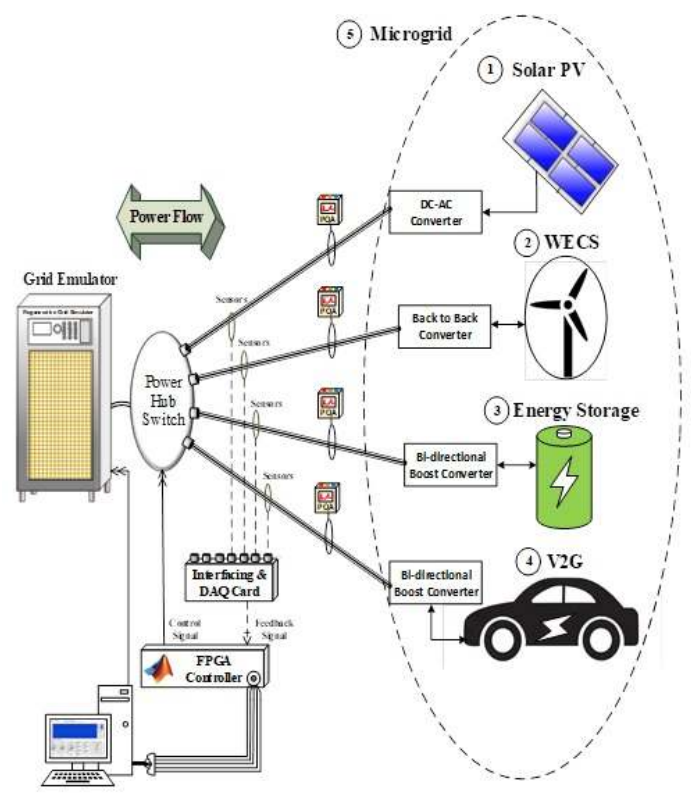

Figure 2. Proposed test rig

ii. Determining ramp-up and ramp-down time constant during input DC variation

The objective of this test method is to file the overall functioning of the inverter during drastic input change as well as the transients (this problem may occur because of the fast shading at the generation of solar site or due to velocity variability in the conversion device for wind energy). The inverter test has to be executed in the following sequences;

- Initially set the steady-state voltage and frequency in regenerative grid emulator and then connect the inverter to it.

- PV simulator is fed the various solar irradiation profiles. Those profiles are checked many times in real time and at higher speeds.

- Feed-in the velocity variation in wind emulator with different pitch angle repeatedly that varies the input of the inverter.

- Analyse the output current/inverter power and find out the behaviour of inverter for different input variations.

- Carryout the test for several times at different speeds of irradiance and different velocity and make sure that time delay should be consistent.

\section{B. Anti-Island test of Grid-tied Power Converters}


Unintended isolation (islanding) of distributed generation can lead to power quality problems, damage to equipment, hazards to personnel safety and interference with grid protection devices. A global analysis of the anti-islanding scheme shows that the existing solutions are too costly, not secure or cause degradation of power quality. The reason behind this test is to analyse the inverter performance under grid connected mode. The transient over voltages of inverter are generated during disconnection of grid which is provided by this test. The inverter test has to be executed in the following sequences;

(i) Initially set the steady-state voltage and frequency in regenerative grid emulator and then connect the inverter to it.

(ii) Inverter is allowed to feed rated power into the regenerative grid emulator.

(iii) Various power quality perturbations including over-voltage transient are created using regenerative grid emulator.

(iv) Set up the power analyser and data acquisition to collect voltage and current in high sampling.

(v) Analyse the inverter behaviour during over-voltage transient and enable the protection circuit as per international standards such as DIN V VDE V 0126-1-1, VDE-AR-N 4105, and IEEE 1547.

(vi) Carryout the test for multiple times by disconnecting the inverter from the grid.

C. Under-voltage and Over-voltage Transient Test

This is to test the inverter output during over voltage and under voltage transients also called as swell and sags (as per IEEE standard) respectively. The voltage transients occurring at the inverters should have " $\mathrm{t}$ " $(\mathrm{t}=3,6,9$, and 12) cycles of duration time which signifies the switching times for some of the circuit breakers. Inverter takes 1 to 3 seconds to get settled down from the voltage changes. In case, if the protection system enable and shutdown the inverter on any of the transients, the restarting takes longer duration of up to 5 minutes. The inverter test has to be performed in the following sequences;

(i) To begin with, connect the inverter to regenerative grid emulator after adjusting the steady-state voltage and frequency.

(ii) Setup the sag or swell with different depth and time interval using regenerative grid emulator. Time to time, power analyser and data acquisition is used to collect inverter voltage and current in high sampling.

(iii) Fix the voltage sag and swell depth constant and start testing with longest duration time. The time taken by the inverter to trip after a transient is measured. Secondly, inverter is tested with lesser duration (which is shorter than the inverter trip time measured previously). Continue the test by reducing the interval, until the inverter stop tripping.

(iv) Repeat the test with different depth and analyse either the output current of the inverter or output power for determining the corresponding voltage magnitude and trip times.

D. Voltage Oscillation Test

This is to examine inverter output during voltage oscillations usually seen during grid disturbances. The amplitude of the inverter output voltage at various envelope frequencies is to be modulated between 90 percent and 100 percent. The inverter test has to be performed in the following sequences;

(i) To begin with, connect the inverter to regenerative grid emulator after adjusting the steady-state voltage and frequency.

(ii) Regenerative grid emulator is programmed for producing the voltage swing. If the desired voltage range exceeds the protective envelopes that cause Switch off the inverter, the voltage profile could be configured over and over again to modulate between smaller ranges. 
(iii) Enable the data acquisition to collect voltage and current in high sampling, and analyse either the output current or output power of the inverter for determining the corresponding voltage magnitudes and trip times.

\section{E. Test on under and Over Frequency Fluctuations}

In this test, evaluation of the inverters behaviour during under and over variations in frequency which are usually seen in the grid or as per the specifications of IEEE 1547. Such frequency variations must have " $t$ " length cycles $(t=3,6,9$, and 12) that serve the switch times for certain common circuit interrupters or circuit breakers. The inverter test has to be performed in the following sequences;

(i) To start with, link the inverter to the regenerative grid emulator after adjusting the steady-state voltage and frequency, and then fed the grid emulator to the inverter with a rated power.

(ii) Implement a frequency transient with different frequency level and switching time using regenerative grid emulator. During any transients if the inverter shuts down, restarting takes longer duration up to, So make sure this time is random or compatible with measurement.

(iii) Fix the frequency sag and swell depth constant and start testing with longest duration time. The time taken by the inverter to trip after a transient is measured. Secondly, inverter is tested with lesser duration (which is shorter than the inverter trip time measured previously). Continue the test by reducing the interval, until the inverter stop tripping.

(iv) Repeat the test with different depth and analyse either output current on the inverter or power to find out magnitudes of the voltage and time of trips.

F. Test on Frequency Oscillation

The main intention of this test is to estimate the behaviour of the inverters at the time of frequency dynamics mostly observed during disturbances on the grid. The frequency of the inverter is set between $51.5 \mathrm{~Hz}$ to $48.5 \mathrm{~Hz}$ at different frequency envelopes. The inverter test has to be performed in the following sequences;

(i) To begin with, connect the inverter to regenerative grid emulator after adjusting the steady-state voltage and frequency and then rated power fed to the inverter.

(ii) Regenerative grid emulator is programmed for producing the frequency swing. If the recommended frequency range exceeds the protection envelope range then it leads trip off of the inverter. Due to this, reprogramming of voltage profile is done between the smaller ranges.

(iii) Enable the data acquisition to collect voltage and current in high sampling, and analyse either output power or output current of the inverter for determining the consequent voltage magnitudes and trip times.

G. Voltage and Frequency Ramp Test

From this test, inverters dynamic performance along with the contact and output values are lies between its (P, I, and Q) at distinct frequencies and voltages. With the data collected, utilization for development of model and validation is done. The inverter test has to be performed in the following sequences;

(i) To begin with, connect the inverter to regenerative grid emulator after adjusting the steady-state voltage and frequency.

(ii) The frequency should be maintained $(50 \mathrm{~Hz})$ throughout the experimentation.

(iii) Regenerative grid simulator is programmed to ramp down/up the system voltage at different time intervals (4sec, $8 \mathrm{sec}$, and $16 \mathrm{sec}$ ) durations from voltage $100 \%$ to $60 \%$, and/or $50 \%$. Under this condition, the inverter should not trip off.

(iv) The voltage should be maintained $(50 \mathrm{~Hz})$ throughout the experimentation. 
(v) Enable the data acquisition to collect voltage and current in high sampling, and analyse either the inverter output current or output power for determining the corresponding voltage magnitudes and trip times.

\section{H. Conservation Voltage Reduction test}

From this test inverter behaviour can be assessed at conservation voltage reduction (CVR), of other utilities in order to minimize the utilization of electrical power via loads from customer. The below mentioned sequences are followed for the inverter to test;

(i) To begin with, connect the inverter to regenerative grid emulator after adjusting the steady-state voltage and frequency.

(ii) Regenerative grid emulator is programmed to reduce the system voltage in steps. The reduction of voltages at every $10 \mathrm{sec}$ is reduced with a $1 \%$ of nominal voltage for better information at different voltage levels.

(iii) Enable the data acquisition to collect voltage and current in high sampling, and analyse either the output current or of inverter for determining the corresponding voltage magnitudes and time of trips.

\section{Harmonic analysis}

Calculation of harmonics generated by this test is done at the inverter under the conditions of steady state. Due to over load on utilization equipment's, unintended negative impacts and high harmonic generation hassle. Total demand distortion (TDD) and Total harmonics distortion (THD) of voltage and current are required. Additionally, the waveform synthesis program technique injects different distorted harmonic waveforms which are required by some regulatory standards. The inverter test has to be performed in the following sequences;

(i) Initially set the steady-state voltage and frequency in regenerative grid emulator and then connect the inverter to it.

(ii) Inverter is allowed to feed rated power into the regenerative grid emulator.

(iii) Harmonic injection is carried out using waveform synthesis program technique from regenerative grid emulator.

(iv) The inverter current and voltage are listed at a high proportion of samplings (one million samples/second to record a harmonics of higher order) while in steady-state condition.

(v) Based on the inverter voltage and output currents, TDD, THD and individual harmonics are calculated.

\section{J. Unbalanced test}

This test is conducted in order to analyse the performance of the inverters voltage transients and/or voltage sags at unbalanced conditions, which are mostly at the grid. The inverter test has to be performed in the following sequences;

(i) Initially set the steady-state voltage and frequency in regenerative grid emulator and then connect the inverter to it.

(ii) Rated power is fed to the Inverter for the regenerative grid emulator.

(iii) Program the regenerative grid simulator for 2, 4, 6, to 10 percent voltage magnitude unbalance.

(iv) Program the regenerative grid simulator for $2,4,6$, to 10 percent voltage phase unbalance.

(v) Analyse the inverter output current or output power to regulate the trip times and voltage magnitudes which are helpful for the voltage protection envelope of inverters, during inverter shutdown

K. Voltage Ride-Through (VRT) test 
To ensure its reliability of Low VRT as per the specified grid codes during different fault conditions is essential. Low VRT characteristics are distinct from various countries with minor changes in voltage recovery time, magnitude of voltage drop, fault time and final voltage magnitude. This test is to find out the ability of the inverters to ride through at low and high voltage conditions, which help the inverter shutdown protection. This test avoids the redundant inverter disconnection from grid which could make events worse of the system. The inverter test has to be performed in the following sequences;

(i) Initially set the steady-state voltage and frequency in regenerative grid emulator and then connect the inverter to it.

(ii) Inverter is allowed to feed rated power into the regenerative grid emulator.

(iii) Generation of Transient voltage is done by Regenerative grid simulator through programming for a fault-induced delayed during the event of voltage recovery.

(iv) Analyse output current or output power of the inverter to find out the respective voltage values and trip times considered in voltage protection envelope of the inverters, where it shuts down.

\section{Inverter Dcperformance Test}

Performance tests on inverter by applying DC are to analyse the performance [14-16] of the inverter especially during the changes in voltage as well as power in the DC.

A. Test on Inverter "DC On" Time Delay

From this test, starting time of the inverter in generating the power (AC) and also its ramp rate when connected to the DC is analyzed.

Step 1: Testing of Setup

a. Switch grid simulator to $\mathrm{ON}$ and it should be confirmed that frequency and voltage are at rated values and before starting of test at inverter, this simulator is connected to inverter.

b. Before starting of the test, Turn ON the PV simulator but with OFF position of its output switch.

Step 2: Switch to the ON position by closing switch of the PV Simulator.

Step 3: Output current/power can be analyzed at the inverter and note down the time taken for the inverter in reaching its rated output

Step 4: Carry out the test more number of times to ensure that there is a steady in delay time.

B. Test on Inverter "DC Off” Delay

In this test, Inverter delay time is determined to shut down the conversion of power upon disconnection.

Step 1: Testing of Setup

a. Switch ON the PV simulator and before testing, inverter need to be connected.

b. Turn $\mathrm{ON}$ the grid simulator and confirm that steady state frequency and voltage are obtained, before starting the inverter test.

c. Before the starting of the test, make sure that power is generating by the inverter.

Step 2: By tuning OFF the PCC circuit breaker, inverter is to be shutdown.

Step 3: Inverter output power/current can be analyzed and also record the time taken for the inverter in reaching its minimum output current.

Step 4: Conduct the test more number of times to ensure the delay time is steady.

C. Test on Irradiance Profile 
The test aims is to record the overall inverter response and transients produced when the changes in PV appears drastically due to a fast solar generation shading.

Step 1: Testing of Setup

a. Turn ON the PV simulator and inverter to be attached until this test starts

b. Turn ON grid simulator and ensure that the frequency and voltage are stable before testing and inverter to be connected.

c. Before this test starts, ensure power is provided by the inverter

Step 2: playback or Feed in into a PV simulator with just an irradiance profile. The profile testing with irradiance may be performed many times and at different speeds.

Step 3: Analyse the inverter's output power/current and assess inverter activity during fluctuations in irradiance.

Step 4: Carry out the test several times with different irradiance speeds for consistent delay time

\section{Performance Tests Of Inverter (AC)}

The aim of these Tests is to assess efficiency of the inverter during changes in voltage and frequency of the AC. Attach the grid simulator, inverter, load bank, and DC source for all of those tests.

A. Test on Time Delay at Inverter Output

This test is to determine the time delay of the inverters when abruptly attached or rejoined to the grid, in particular its grid-generating power.

Step 1: Testing of Setup

a. Turn ON PV simulator and attach to the inverter until this test starts.

b. The frequency and voltage of the grid simulator should remain stable and not be connected to the inverter before the test.

c. Before starting this test, ensure that the inverter output switch is OFF (open).

Step 2: Turn AC switch to ON position to power the inverter.

Step 3: Inverter's output current / power is analyzed and find out time of inverter to reach their nominal output.

Step 4: Repeat the test several times to ensure accuracy of the delay time.

B. Test on Inverter Anti-Island

This test is to assess the performance of anti-islanding of the inverters. This test reveals the transient overvoltage's generated by the inverters during disconnection of grid.

Step 1: Testing of Setup

a. Before this test begins the PV simulator should be ON.

b. The voltage and frequency of the grid simulator should remain stable before the test and during the test.

c. Connect power analyser to collect high currents and voltages.

d. Inverter will produce complete power over the grid

Step 2: By disconnecting the inverter from the grid, perform several examinations.

Step 3: Analyse the inverter-generated transient over-voltage along with the voltage across the breaker terminals which are opened during the test. 
Table 1.Anti Islanding Test

\begin{tabular}{llllll}
\hline \multirow{2}{*}{ Test } & Load 1 & \multicolumn{2}{l}{ Load 2 } & Inverter \\
\cline { 2 - 6 } & KVA & pf & KVA & pf & pf \\
\hline 1 & $150 \%$ & 0.8 & $0 \%$ & 0.8 & 1 \\
\hline 2 & $125 \%$ & 0.8 & $25 \%$ & 0.8 & 1 \\
\hline 3 & $100 \%$ & 0.8 & $50 \%$ & 0.8 & 1 \\
\hline 4 & $75 \%$ & 0.8 & $75 \%$ & 0.8 & 1 \\
\hline 5 & $50 \%$ & 0.8 & $100 \%$ & 0.8 & 1 \\
\hline 1 & $150 \%$ & 0.8 & $0 \%$ & 1 & 1 \\
\hline 2 & $125 \%$ & 0.8 & $25 \%$ & 1 & 1 \\
\hline 3 & $100 \%$ & 0.8 & $50 \%$ & 1 & 1 \\
\hline 4 & $75 \%$ & 0.8 & $75 \%$ & 1 & 1 \\
\hline 5 & $50 \%$ & 0.8 & $100 \%$ & 1 & 1 \\
\hline 1 & $150 \%$ & 0.8 & $0 \%$ & 0.8 & 0.8 \\
\hline 2 & $125 \%$ & 0.8 & $25 \%$ & 0.8 & 0.8 \\
\hline 3 & $100 \%$ & 0.8 & $50 \%$ & 0.8 & 0.8 \\
\hline 4 & $75 \%$ & 0.8 & $75 \%$ & 0.8 & 0.8 \\
\hline 5 & $50 \%$ & 0.8 & $100 \%$ & 0.8 & 0.8 \\
\hline 1 & $150 \%$ & 0.8 & $0 \%$ & 1 & 0.8 \\
\hline 2 & $125 \%$ & 0.8 & $25 \%$ & 1 & 0.8 \\
\hline 3 & $100 \%$ & 0.8 & $50 \%$ & 1 & 0.8 \\
\hline 4 & $75 \%$ & 0.8 & $75 \%$ & 1 & 0.8 \\
\hline 5 & $50 \%$ & 0.8 & $100 \%$ & 1 & 0.8 \\
\hline & & & & & \\
\hline
\end{tabular}

\section{Test on Under-Voltage Transients}

This test objective is to determine the inverter efficiency at the time of transient voltage sags and/or under voltages that's usual of the standard safety requirements of the electrical grid and/or IEEE. In order to let the inverter stabilize, voltage sags of the above must have interval of 1 to 3 seconds between changes in voltage. These different duration times for the sag voltage $(t=3,6,9$, and 12 cycles) constitute triggering times on all circuit breakers which are common. Additional tests $(\mathrm{t}=10,110,120$, and 130 cycles) reflect the requirements of IEEE 1547 security. The test of $\mathrm{t}=1$ cycle, is a rapid transient produced by equipment which performs switching action. The transient test under voltages is shown in Figure. 3

Step 1: Testing of Setup

a. Before the test starts, turn $\mathrm{ON}$ the PV simulator and inverter should be connected to it.

b. The frequency of the grid simulator should remain constant at $60 \mathrm{~Hz}$ as well during testing.

c. Before the test starts, the voltage to the grid simulator should be stable and also inverter connected it.

d. The inverter must generate full power inside the grid

e. On the inverters, the 100 percent of rated power must be achieved at the load bank.

Step 2: Transient voltage is implemented and switching states of the Grid simulator is $100 \%$ voltage to $\varepsilon \mathrm{V}$ and $\varepsilon$ vice-versa, should be attained as fast $(\sim 0.2 \mathrm{~ms})$ as possible. If the inverter turns off on a transient, it can take as much time as 5 minutes to restart. 


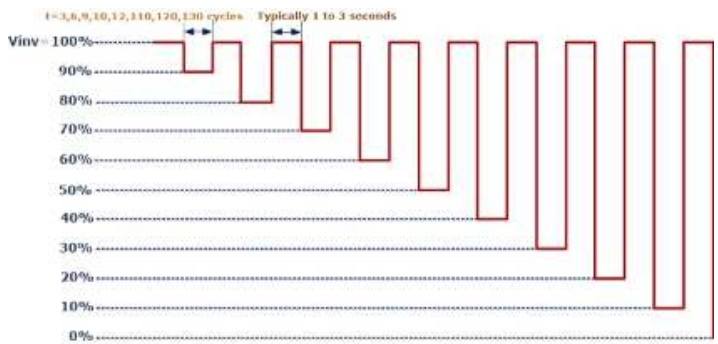

Figure 3. Under Voltage transient

Effective way of testing is to begin with a full range and then calculate the time taken for the inverter to travel subsequent to a transient. In the next comparison, the usage of a voltage sag length period is only shorter than the inverter trip time calculated beforehand. This would minimize research over excessive lengthy periods. In the event that this test fails due to voltage/current not fully recovering from a stable- state till the next sag, the transients of under voltage can need to be alone achieved as stated in Step 3, then skip to Step 4.

Step 3: Lower the voltage at the output of a grid simulator $(\mathrm{\varepsilon V})$ at $\mathrm{t} 1$ and raising it at $\mathrm{t} 2$ to nominal voltage as shown in Figure 6. Begin with the voltage of the sag $(\varepsilon \mathrm{V})$ level at 90 percent and rising it in decreases by 10 percent before device is shut down.

Step 4: Output Power of Inverter or Output current of Inverter are analyzed to assess the respective magnitudes of voltage, trip times, and the inverters' voltage safety envelope. The transient voltage is shown in Figure. 4

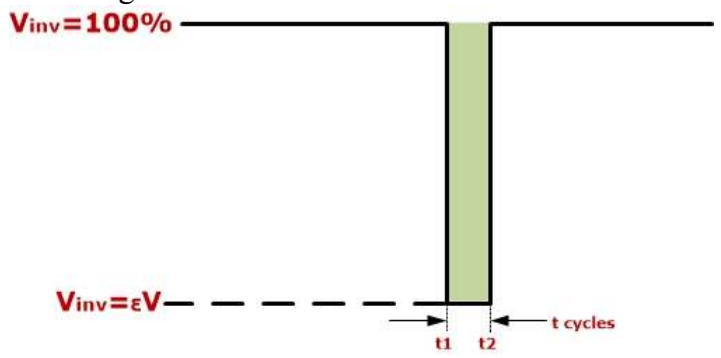

Figure 4. Voltage transient

D. Test on Over-Voltage Transients

This test aims in evaluating the inverter output typical of the electrical grid and/or standard of IEEE safety requirements during voltage swells and/or over-voltage transients. In order to let the inverter relax, these voltage swells would have " $t$ " length cycles and with time of 1 to 3 seconds span among voltage shifts. These various period periods of swell voltage $(\mathrm{t}=$ $3,6,9$, and 12 cycles) which are seen in some common circuit breakers, reflecting their switching times. The further tests $(t=10,50,60$, and 70 cycles) reflect the specifications of IEEE 1547 security. The $\mathrm{t}=1$ cycle test is a rapid transient generated through switching equipment. The transient test for over voltage is seen in Figure. 5. 


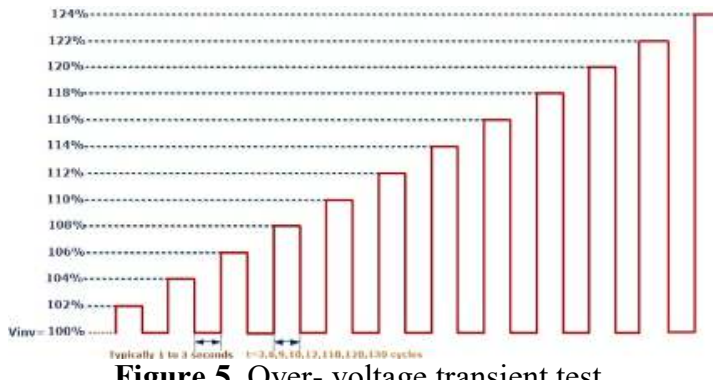

Step 1: Testing Setup

Figure.5. Over- voltage transient test

a. Before the test starts turn ON Connect PV simulator with inverter

b. The frequency of grid simulator should continue stable at $60 \mathrm{~Hz}$ before and during testing.

c. Before the test starts, a stable voltage should be at grid simulator and should also be connected by an inverter.

d. The inverter must generate maximum power inside the grid

e. Load bank be 100 percent of the rated capacity of the inverters.

Step 2: Transient voltage is implemented which are related to that indicated in Figure 5. Switching of the grid simulator, $100 \%$ voltage to $\mathrm{V}$ and vice-versa must be implemented approximately $0.2 \mathrm{~ms}$. When the inverter falls off due to any transient, restoring can take around 5 minutes. Most convenient way to carry out this test is it should begin with the greatest possible period, and then measuring the time taken for the inverter to take trips after such a transient. Using duration of the voltage sag only shorter than the ones calculated before trip time on the inverter for the next test. This avoids testing over unnecessary times of duration. When this test is unsuccessful due to current/voltage not recovering completely before the next swell, the transient overvoltage might be individually executed as mentioned in Step 3, if not skip to Step 4.

step 3: Grid Simulator Output voltage should be raised to t1 swell voltage $(\varepsilon \mathrm{V})$ and fall down to the $\mathrm{t} 2$ voltage of nominal value. The grid simulator starts at 100 percent and increases by 2 percent in increments before they reach the unit 124 percent or before inverter shuts down.

Step 4: Evaluate the current / power of the output of the inverter for the determination of the respective voltage magnitudes and time of trips, the voltage protection envelope of the inverters.

\section{i. Test on Voltage-Oscillation}

Determination of the output of the inverters at the time of voltage oscillations normally observed on the grid at the time of disturbances is obtained by this test. The change in amplitude of the inverter output voltage at different envelope frequencies between $100 \%$ and $90 \%$. The frequencies in the sample envelope ( $\mathrm{f}(\mathrm{swing})=0.1,0.25,0.7,1,2 \mathrm{~Hz}$ ) shown in figure 6 , gives the oscillations of the grid. 


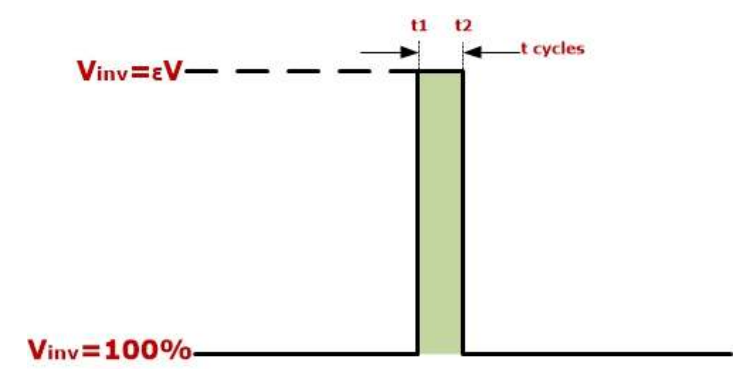

Step 1: Testing of Setup

Figure.6. Voltage transient

a. Before the test starts, turn ON Connect PV simulator with inverter

b. The frequency $(60 \mathrm{~Hz})$ of the grid simulator should remain stable before and testing period.

c. Before the test starts, a stable voltage should be at grid simulator and should also be connected by an inverter.

d. The inverter must generate rated power inside the grid

e. Load bank shall be 100 percent of the inverters rated capacity.

Step 2: Grid simulator Voltage to emulate the behaviour must be programmed.

Step 3: Current / power balance of the inverter to find out the respective voltage magnitudes and trip times need to be analyzed.

ii. Test on Under-Frequency Fluctuations

Evaluation of the efficiency of the inverters during under frequency fluctuations normally found in the standard security requirements of the electric grid and/or IEEE. For letting the inverter to settle, voltage sags should have t cycles for a time period of 1 to 3 seconds of interval between the changes in voltage. These distinct times under frequency durations $(t=3$, 6,9 , and 12 cycles) for some regular circuit breakers represent switching times. The further tests $(t=10,110,120$, and 130 cycles $)$ reflect the requirements of IEEE 1547 security. The test $\mathrm{t}=1$ cycle, is a rapid transient occurred through equipment of switching. It is depicted in figure 7 under frequency observation.

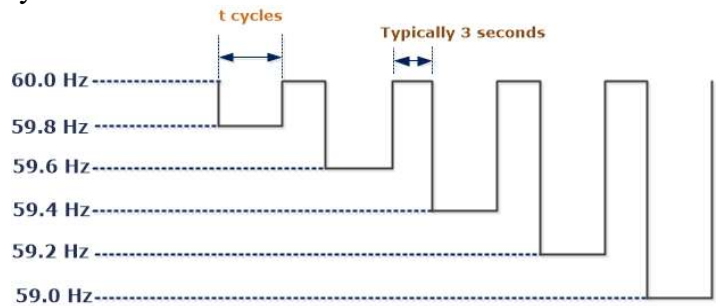

Figure. 7. Under Frequency Fluctuation test

Step 1: Testing of Setup
a. Before the test starts turn ON Connect PV simulator with inverter
b. The voltage should be stable across the grid simulator at the time of test
c. Rated power should be generated by the inverter inside the grid
d. Load bank be 100 per cent of the rated power of the inverters

Step 2: Similar transient frequency is implemented. Grid simulator switch times must be done soon as possible $(\sim 0.2 \mathrm{~ms})$ between the different frequency levels. Due to any transient, if inverter off and it may require g5 minutes to restart; make sure to assess whether this time is random or consistent. 
If this test is ineffective because the current does not fully recover to a steady state until the next sag, the underfrequency transients will have to be carried out individually, as stated in Step 3, else skip to Step 4.

Step 3: The frequency of the grid simulator must be reduced to the frequency of a sag $(\varepsilon \mathrm{Hz})$ of $\mathrm{t} 1$ and returned at $\mathrm{t} 2$ to $60 \mathrm{~Hz}$. The sag frequency $(\varepsilon \mathrm{Hz})$ could start at $59.8 \mathrm{~Hz}$, then decrease it by $0.2 \mathrm{~Hz}$ until the unit shuts down.

Step 4: Analyse the output current/power of the inverter to find out the corresponding trip times and frequency values which are utilised for the inverters frequency protection envelope.

\section{iii. Test on Over-Frequency Fluctuations}

This test is to analyse the inverters efficiency, usually found in the electrical grid and/or standards of IEEE safety requirements during over-frequency fluctuations. In order to let the inverter settle, these voltage sags should have $t$ duration cycles and 1 to 3 seconds time of interval between changes in voltage. In some common circuit breakers, the different over frequency durations $(\mathrm{t}=3,6,9$, and 12 cycles) reflect switching times. The additional tests $(\mathrm{t}$ $=10,110,120$, and 130 cycles) reflect the requirements of IEEE 1547 protection.

Step 1: Testing of Setup
a. Before this test starts the PV simulator should be ON
b. The voltage should be stable across the grid simulator at the time of test
c. The inverter must generate rated power inside the grid
d. Load bank be 100 per cent of the rated power of the inverters

Step: 2 Grid simulator switch times must be accomplished approximately at $0.2 \mathrm{~ms}$ between the different frequency levels. If the inverter off for any transient, it takes 5 minutes to restart; make sure to assess whether this time is random or consistent.

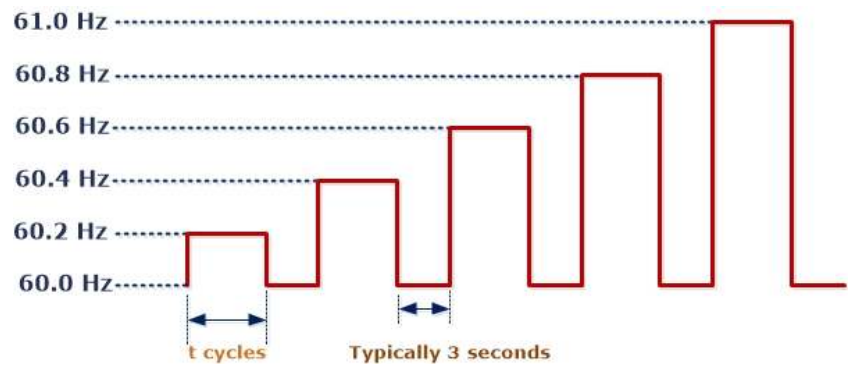

Figure. 8. over frequency fluctuation test

If suppose this test is ineffective because the current does not fully recover to a normal state until next swell, then the over frequency transients need to be carried out individually, as stated in Step 3, else go to Step 4.

Step 3: Grid simulator frequency can be raised to t1's swell frequency $(\varepsilon \mathrm{Hz})$ and then dropped back to t2 at $60 \mathrm{~Hz}$. The frequency of swell $(\varepsilon \mathrm{Hz})$ is introduced which starts at 60.2 $\mathrm{Hz}$ and raising it in increments of $0.2 \mathrm{~Hz}$ before shut down of unit.

Step 4: Analysis of the current / power inverter output to evaluate the trip times and the corresponding frequency values that are used for the safety of frequency envelope inverters. Above fluctuation test is shown in figure.8.

iv. Frequency Oscillation Test

This test is to assess the behaviour of the inverters during frequency oscillations typically observed on the grid at the time of disturbances. Modulation of inverter frequency is to be done at different envelope frequencies between $61 \mathrm{~Hz}$ and $59 \mathrm{~Hz}$. The frequencies in the sample envelope $(\mathrm{f}(\mathrm{swing})=0.1,0.25,0.7,1,2 \mathrm{~Hz})$ represents grid oscillation .

Step 1: Testing of Setup 
a. Before this test starts the PV simulator should be $\mathrm{ON}$

b. The voltage should be stable across the grid simulator at the time of test

c. The inverter must generate rated power inside the grid

d. Load bank be 100 per cent of the rated power of the inverters

Step 2: Program the frequency of the grid simulator to replicate the behaviour.

Step 3: Analyse inverter output current / power, as it corresponds to the oscillating system frequency.

\section{v. Test on Voltage Ramp}

This test is to know the complex behaviour of the inverters at different voltages which includes the relation between its output (Q, P, and I) values. Later, the collected data used to develop and validate models.

Step 1: Testing of Setup

a. Before this test starts the PV simulator should be ON

b. The frequency of the grid simulator should remain stable at $60 \mathrm{~Hz}$ before and at the time of the test

c. Before the test starts, the voltage across grid simulator must be stable and it is inverter connected.

d. Inverter should generate rated power inside the grid

e. Load bank be 100 per cent of the power rated by the inverters

Step 2: Grid simulator must be programmed to ramp down and ramp up the voltage of the system from 100 percent to 90,80 , and/or 50 percent voltage in 4,8 , and 16 seconds, provided the inverter does not leave.

Step 3: Analyse inverter output current / power and its behaviour in response to system voltage changes.

\section{vi. Test on Frequency Ramp}

This test is to know the complex behaviour of the inverters at different frequencies which includes the relation between their output values $(\mathrm{Q}, \mathrm{P}$, and I). Later, the collected data used for validation of the model. Test of the frequency ramp is shown in Figure $9 \& 10$.

Step 1: Testing of Setup

a. Before this test starts the PV simulator should be ON

b. The frequency of the grid simulator should remain stable at $60 \mathrm{~Hz}$ before and at the time of test

c. Before the test starts, the voltage across grid simulator must be stable and it is inverter connected.

d. Inverter should generate rated power inside the grid

e. Load bank be 100 per cent of the power rated by the inverters

Step 2: Grid simulator must be programmed to ramp down and ramp up the frequency of the system in 4,8 , and 16 seconds lengths from $60 \mathrm{~Hz}$ to $\varepsilon \mathrm{Hz}$, provided the inverter doesn't exit.

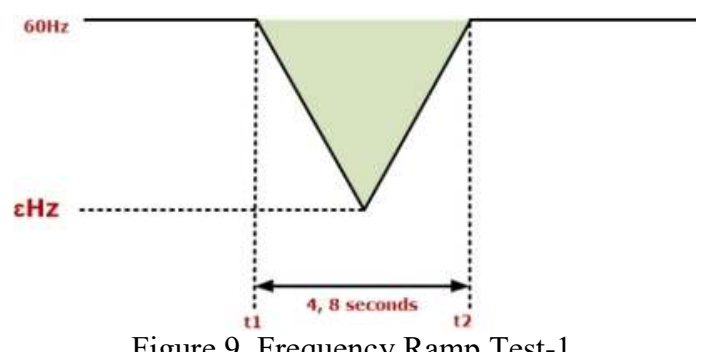

Figure.9. Frequency Ramp Test-1 
Step 3: Analysis of current / power inverter output and its behavior in response to system frequency changes.

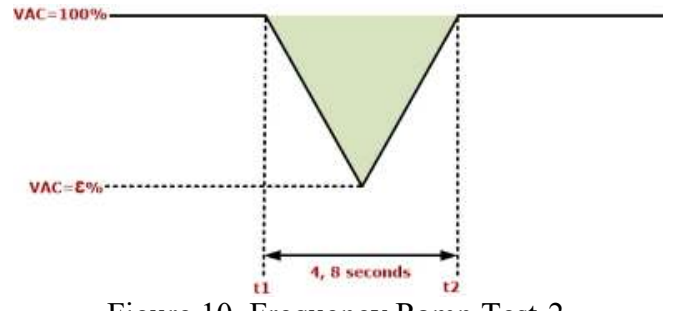

Figure.10. Frequency Ramp Test-2

\section{vii. Test on Conservation Voltage Reduction (CVR)}

This test is to evaluate the behaviour of the inverter at the time of conservation voltage reduction (CVR), automized by some utilities to lower the power consumption by customer loads.

Step 1: Testing of Setup

a. Before this test starts the PV simulator should be ON

b. The frequency of the grid simulator should remain stable at $60 \mathrm{~Hz}$ before and at the time of test

c. Before the test starts, the voltage across grid simulator should be stable and inverter connected.

d. Inverter should generate rated power inside the grid

e. Load bank be higher than $100 \%$ of the power rating inverters

Step 2: Grid simulator must be programmed to decrease the voltage of the network. In Figure 11 CVR is represented.

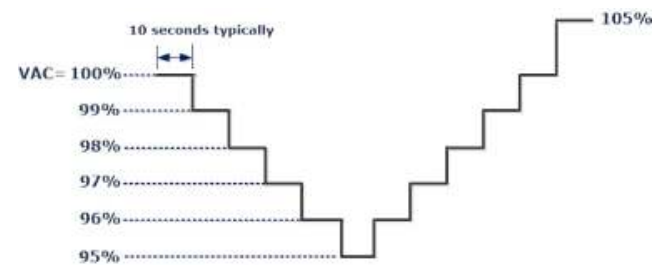

Figure.11. Conservation Voltage Reduction

Step 3: Analysis must be done to the output of current / power of the inverters and their behaviour in response to the system voltage changes.

\section{viii. Test on Data Recording of Harmonics}

This test is to evaluate the inverter-generated harmonics under stable state conditions. By overloading, high harmonic generation may cause Unintended serious negative consequences on utility field equipment such as transformers, capacitors and switching equipment. Needed information for both voltages and currents will be total demand distortion (TDD) and total harmonic distortion (THD).

Step 1: Testing of Setup

a. Before this test starts the PV simulator should be ON

b. The frequency of the grid simulator should remain stable at $60 \mathrm{~Hz}$ before and at the time of the test

c. The stable voltage across grid simulator must be maintained and is connected to the inverter before and at the time of test.

d. Inverter should generate rated power over the grid 
e. Load bank be higher than 100 per cent of the power rated by the inverters

Step 2: Note the current and voltage of the inverter at a sampling rate of higher while in permanent state. It is proposed that data of 1 second should be collected at a rate of 1 million samples per second to obtain a harmonics of higher order

Step 3: Analysis of the inverter current and voltage current for measuring the THD, individual harmonics and TDD

\section{Three Phase Inverter Ac Performance}

When explicitly evaluating the efficiency of 3-phase solar PV inverters, the following tests shall be carried out referring from Fig $12 \& 13$.

i. Test on Unbalanced Under-Voltage Transients

This test is to know the behaviour of the inverters at the time of unbalanced low voltage transients and/or voltage sags typical of the electrical grid

Step 1: Testing of Setup

a. Before this test starts the PV simulator should be ON

b. The frequency of the grid simulator should remain stable at $60 \mathrm{~Hz}$ before and at the time of the test

c. Before the test starts, the voltage across grid simulator must be stable and inverter connected.

d. Inverter should generate rated power inside the grid

e. Load bank be 100 percent of the power rated by the inverters

Step 2: Output of Grid simulator line to neutral voltages can be reduced at t1 to voltage sag $(\varepsilon \mathrm{V})$ and in various combinations rise back to fundamental voltage at $\mathrm{t} 2$. The level of the sag voltage is achieved by the grid simulator originating at $90 \%$ and decreased by $10 \%$ before the device is shut down. Voltage sag period times $(\mathrm{t}=3,6,9$, and 12 cycles $)$ the reflect time of switching for some common circuit breakers and $(\mathrm{t}=10,110,120$, and 130 cycles) are the security requirements of standard IEEE 1547.

Step 3: Analyse current / power inverter output to determine the corresponding trip times and voltage magnitudes utilized for the voltage safety envelope inverters.

ii. Over-Voltage Transients (Unbalanced) Test

This test is to evaluate the inverter efficiency at the time of transients (unbalanced) of overvoltage and/or swells typical of the electrical grid.

Step 1: Testing of Setup

a. Before the test starts the PV simulator should be $\mathrm{ON}$ and inverter connected

b. The frequency of the grid simulator should remain stable at $60 \mathrm{~Hz}$ before and at the time of the test

c. Voltage of grid simulator must be in stable condition and inverter connected before the test starts

d. Inverter must generate rated power inside the grid

e. Load bank must exceed 100 percent of the power rated by the inverter

Step 2: Grid simulator line to neutral output voltages should be increased to t1's swell voltage $(\varepsilon \mathrm{V})$ and dropped back to t2's nominal voltage in the different combinations found in Figure 5.2.1. The grid simulator starts at 100 percent, and increases by 2 percent in increments until the unit meets 124 percent, or the inverter trips off. The voltage sag is $(t=3,6,9$, and 12 
cycles) representing switching times for some common circuit breakers and $(\mathrm{t}=10,110,120$, and 130 cycles) representing the requirements for protection of standard IEEE 1547.

Step 3: Analyze the output current / power of the inverter to find the corresponding magnitudes of voltage and trip times used for the protection of voltage envelope of the inverters.

\section{Challenges And Issues In Grid Connected Pv Inverters}

There are two types of issues related to PV systems / Technical problems and contractual issues are involved.

\section{A. Technical Issues}

\section{i. Safety}

Recently research programs have been under way to find ways of reducing intrinsic health risks interconnected with PV devices [31-33]. More lately, industrial customers have concentrated on PV generation which are interconnecting systems; work in shunted with the grid. Extensive security gear and periodic inspections by professional/utility engineers can be substantial and accessible for the protection of enormous generation sites, but such demands are unrealistic for low photovoltaic systems.

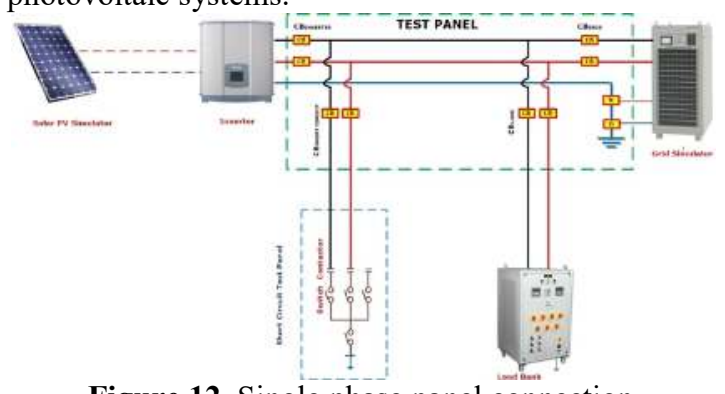

Figure.12. Single phase panel connection 


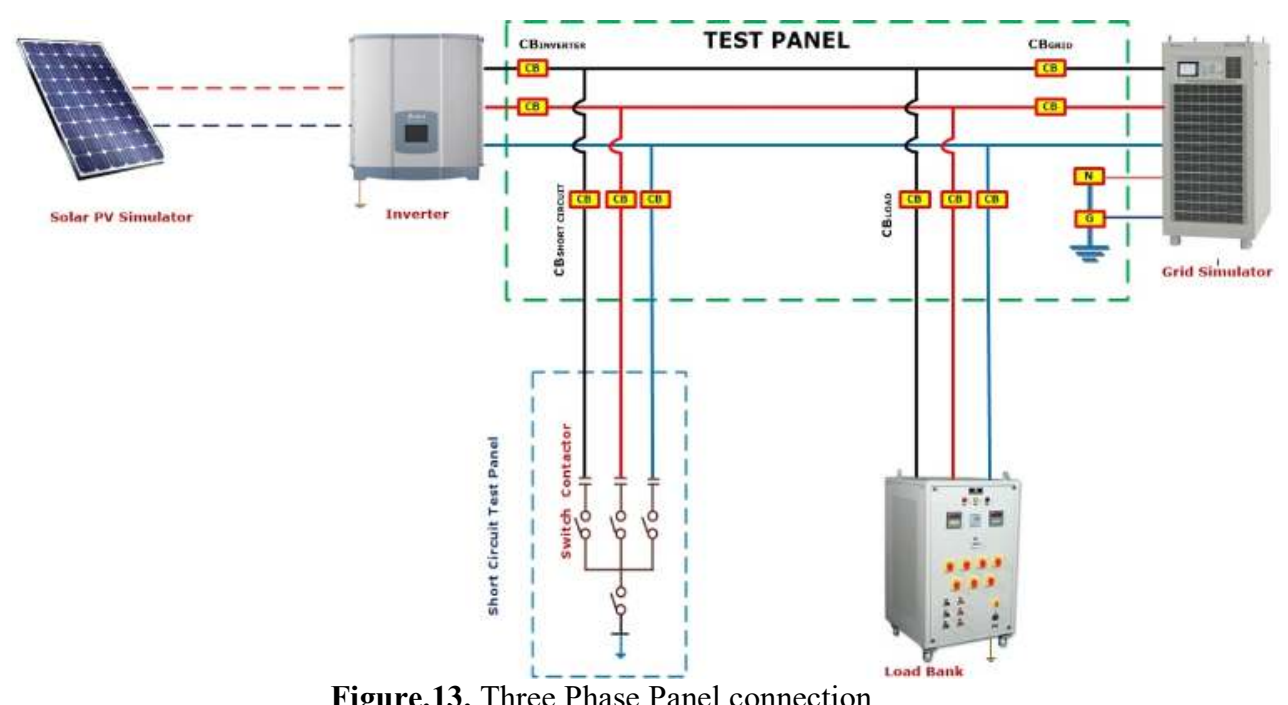

Figure.13. Three Phase Panel connection

Based on the architecture of the system, few connected PV systems to the utility work at DC voltages above $300 \mathrm{~V}$ until being reversed to rated AC current. DC's potential fire hazard at all particular voltages it's indeed larger than the standard $\mathrm{AC}$, because a $\mathrm{DC}$ arc is harder to extinguish than an arc of AC at similar voltage. Proper cabling, however helps to ensure such a hazards pertaining to DC power are reduced significantly.

\section{B. Islanding}

A condition called islanding is among the most critical safety problems for small customer PV systems. Islanding happens when a part of the utility grid containing both generation source and load are seperated from the rest of the utility grid also continue to be energized. By using a PV system if this situation occurs, it is considered to as insulation that is PV accomplished. The safety issue is that, while a utility may ensure that its generating sources are either disconnected/shut down from the region which has to operate, an island generated by the PV systems is out of reach. A worker in utility line may switch in connection with a line which is accidentally energized as a potentially unwanted result of islanding. Inverters with built-in safety features for the anti-insulation are therefore necessary. Inverters of Grid-tied, supervise the utility line and may shut down soon around 2 seconds or less, in case of malfunctions on the utility system.

C. Traceability and Identification:

Use of RF identification tag is required in solar power project for every module of PV but must be capable to endure extreme atmospheric conditions inside or outside the laminate). The Subsequent information must be included in the RFID used on each module:
a. Manufacturer of PV module
b. Manufacturer of solar cell
c. Unique module Model and Serial Number.
d. Manufacturing (for Solar modules and cells separately) month and year
e. Origin State (for Solar modules and cells separately))
f. Module V-I curve
g. Maximum current (Im), Wattage, Fill factor ( FF) and Maximum Voltage $(\mathrm{Vm})$ for the module 
h. Year and date of receiving Standard Certificate of Qualification for PV module

i. Test laboratory details, which issues standard certificates

j. Other applicable details regarding traceability of modules and solar cells according to ISO: 9000

D. Reporting and Measurement

Both grid and solar photovoltaic power plants must be installed with the required facilities to regularly measure ambient temperature, wind speed, solar radiation and other weather parameters and simultaneously calculate the generation of the plant's AC and DC electricity. They are expected to periodically send these data online and/or via a report to the Ministry of Energy. Appropriate measuring facility can be situated as near to the solar plants as possible. The plants located within a five kilometre radius from this facility may use a common measuring facility. This is conditional upon the following:

a. The developers who participate certify that the elevation of the place at this distance range remains similar;

b. The project developers decide on data sharing, facilities management, data quality checks, etc.

E. Power Quality

Other technical concerns for customer-generators and utilities are power quality. Power must be provided at a standard frequency and voltage consistently, since appliances and devices are built to take power at/near the specific location frequency and voltage parameters, and deviations can cause damage/malfunction to the appliances. Problems with power quality can monitor in lines on a TV screen or in static noise on a radio that is often found during the service of a hand mixer or microwave oven. A PV inverter has the potential to inject noise that can cause problems. The power quality requirements include power factor, harmonics, DC injection, and voltage flicker as well as basic voltage and frequency ranges.

a. Generally Harmonics refer to current waveforms and voltage distortions. Such variations are induced by the interference with waves at certain frequencies of the regular $50 \mathrm{~Hz}$ waves. Sinusoidal wave harmonic, which is an integral multiple of the frequency wave and total harmonic distortion (THD) sums all distortions at different harmonic frequencies.

b. Power factor, which is the ratio of real electric power (kilowatts), to the apparent power (kilovolt-amperes), must be brought near unity. This also adds to the otherwise to inefficiencies in the utility system. DC injection occurs when unwanted DC current passes through an inverter into the AC or Inverter Output Side.

c. Voltage flicker refers to short existing spikes/dips in line voltage. A regular manifestation of voltage flicker is the momentary light dimming and Interactive grid inverters don't mostly create problems with voltage flicker or DC injection.

F. Contractual Issues

Although the technical interconnection barriers recently were fairly solved IEEE Std update. 929-2000, UL Std. 1741, considerable contractual barriers to interconnection still remain. [3] These include the requirements for liability insurance, fees and charges etc.

i. Liability

In case of any incidents due to the service of the customer's PV network, liability insurance is provided to cover the utilities and their employee's indemnity is another issue relating to liability which assign to protection against or compensation for harm, injury or loss. When it comes to contracts between photovoltaic owners and utilities, utilities often allow the PV owner or other customer generator to pay the utility for any possible losses arising from 
operation of the PV network. Where protection against liability requirements, exist the requirements for identification are slightly redundant.

\section{ii. $\quad$ Fees and Miscellaneous}

There exist a number of fees levied by utilities can charge on grid-tied PV systems operators. Such costs include charging penalties, fees and charges associated with interconnection, standby charges and metering penalties. Even putting a nominal fee can change the economies of PV systems which are grid-tied considerably.

\section{iii. Agreement Standards and Procedures}

Most critical connectivity problems for customers interconnecting their PV systems are the uniform agreement sector. Even when the main technological and contractual rules are resolved, cost rises and plans are scrapped if advocate need to read and understand the paperwork provided by the company.

\section{Configurations Of Pv Inverter}

In Literature [34-35], there exist distinct types of PV array configurations like, parallel, series, bridge linked, honeycomb and etc. Out of these, the most frequently preferred architecture are the parallel or series and series connections. Series connection of PV Panels is termed as "string" and the same PV panels are connected in parallel, then those are termed as the "Array". Mostly, the PV modules are aligned in four different types such as Central Inverter, Multistring Inverter, String Inverter and Module Integrated or Ac module Inverter as shown in Figure 14.

\section{i. Central Inverter}

In this type of configuration, series connection of numerous panels which forms a string are done for avoiding the amplification of voltage. Moreover these strings are connected in parallel which forms an array in order to enhance the power level. Figure 16, represents the grid connected parallel/series connected PV panels through the central inverter (central structure). A DC-DC boost converter is preferred to step-up the voltage at the PV output under any shaded conditions or cloud cover of the PV panels and then it is connected to the PV inverter for conversion. Panels mismatching is one of the main drawbacks of the central inverter due to the use of a single MPPT for the entire system. Another drawback is that, when central inverter failed to operate due to any issue then the entire PV system operation gets interrupted. Advantage of this central Inverter is overall cost is less when compared to remaining configurations.

\section{ii. String Inverter}

Most frequently and commonly used Inverter is string type. In this string Inverter configuration, inverter connection of each string is done independently such that it avoids the usage of string diode. The problems like mismatching of panels and DC-DC converter usage in central inverter can be eliminated with an seperate MPPT which is attached to each and every string thereby increases the overall efficiency. Range of string configuration is around $5 \mathrm{~kW}$ per string and can be easily expanded for higher ratings. Another issue in the central inverter is that, if one inverter fails to function then the entire system gets interrupted and this issue can be eliminated due to independent connection of strings. Advantages of string inverter are high in reliability, flexibility, less cost of cable and less switching losses. But overall configuration cost are high when compared to the central inverter configuration. 


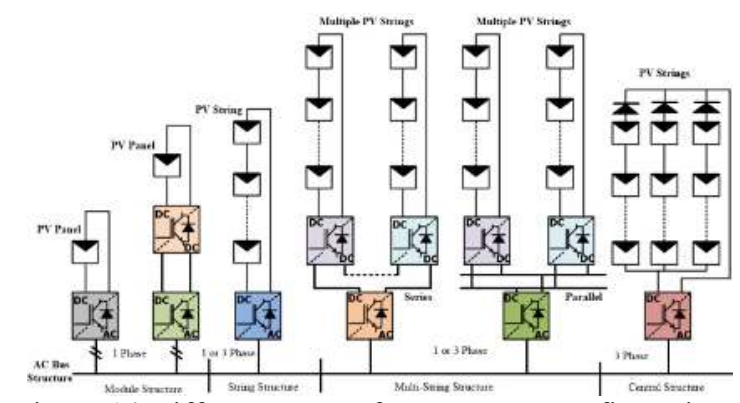

Figure.14.Different types of PV Inverter configurations

\section{i. Multi-String Inverter}

It is a combination of central and string model advantageous and beneficial model. As voltage amplification and use of individual MPPT are seen in this hybrid configuration. Integration of PV strings in this topology can be done and this configuration can also be easily expandable for higher power ratings as discussed in string inverter. These multi-string configurations are costlier when matched to the central structure but are cheaper when matched to $\mathrm{AC}$ module or Module integrated configuration. The range of this multi-string inverter is around $50 \mathrm{~kW}$.

\section{ii. Module Integrated (structure) or AC Module}

This type of configurations is less in size, lower power rating, and also called the microinverter which are mostly preferred in lower powered applicances. In this module, all the operations like, voltage amplification, MPPT and power conversion are done using a single device called the module which are mostly preferred in low power applications. Mismatch losses also can be eliminated due to the connection of MPPT controller in PV module. Table 2 represents the comparative analysis of the central structure, string, multistring and $\mathrm{AC}$ module performance evaluation are tabulated.

Table 2.Comparative Analysis of Inverter configurations

\begin{tabular}{|c|c|c|c|c|c|}
\hline S.No & Parameter & Central & String & Multistring & $\begin{array}{l}\text { Module } \\
\text { Integrated }\end{array}$ \\
\hline 1 & $\begin{array}{l}\text { Variation in } \mathrm{DC} \\
\text { Voltage }\end{array}$ & $\begin{array}{l}\text { Very } \\
\text { High }\end{array}$ & Medium & High & Very Low \\
\hline 2 & Balanced Voltage & High & Medium & Low & Low \\
\hline 3 & Voltage Variation-AC & Low & High & Medium & Very High \\
\hline 4 & Power Loss-AC & Low & Medium & Medium & High \\
\hline 5 & Power Loss-DC & High & Low & Medium & Very Low \\
\hline 6 & MPPT Efficiency & Low & High & Medium & Very High \\
\hline 7 & Reliability & Low & High & Medium & Very High \\
\hline 8 & Flexibility & Low & High & Medium & Very High \\
\hline 9 & Robustness & High & Low & Medium & Very Low \\
\hline 10 & Mismatching & High & Low & Low & Very Low \\
\hline 11 & Switching & High & Low & Medium & Very Low \\
\hline 12 & Cost of Installation & Medium & High & Medium & Very High \\
\hline 13 & Cost of Cables-DC & High & Low & Medium & Very Low \\
\hline 14 & Cost of Cables-AC & High & Medium & Medium & High \\
\hline
\end{tabular}




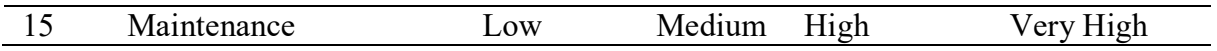

\section{Different Topologies Used In Grid Tied Pv Inverters}

From Figure.15, it represents the single and three phase topologies of PV Inverter. Out of the different inverter configurations as described in section 8, the most commonly preferred configuration is Central structure for obtaining the combined output by series and parallel configurations in major solar powered plants [36-39]. This central structure inverter topologies are most often depends on two level (2L)/ three level (3L) configurations like conventional HBridge (H4), Neutral point clamped (NPC), Voltage source Inverter (VSI) or T-type as shown in Figure 15. The main purpose of using the topologies of inverter is the requirement of single DC bus, that can be easily achieved by coupling of PV power. Inverter output stage comprises of Line frequency (LF) transformer of three phases which reduces the losses and improves the voltage of low value (LV) for the connection to voltage at medium value in distribution lines.Usually the solar inverters consists transformer of LF or High frequency (HF) for voltage transformation ratios or galvanic isolation. Increase of voltage in DC link up to four or five times at the DC-DC converter output side which are supported by single PV module can be achieved by using HF transformers. HB zero voltage rectifier (HB-ZVR), zero-state mid-point clamped topologies oH5, H5 and HERIC topologies are effective in eliminating the leakage currents and are extensively utilized in micro inverters.

\section{Conclusion And Future Work}

Being one of the innovations that are emerging to address the rising demand for energy and the need to reduce carbon dioxide emissions, solar PV and deplete fossil fuels has acquired outstanding importance. Photovoltaic inverter is the cause for energy conversion when using photovoltaic solar power systems. It is crucial to assess the behaviour of the PV inverter which is utilized in the field to assure the reliability and quality of the generation system. As per the IEEE 1547 standards a complete review on the test instructions, islanding and power quality especially voltage, frequency, Harmonics, DC offset, etc. in a step by step procedure are explained clearly which helps in further developments.

Global energy status of the photovoltaic industry in this paper discusses reviews and explains the Categorization of the PV system, i.e. stand-alone grid-connected topologies, gridconnected PV inverter configurations, Inverter types classification, different topologies inverter, control methodologies for single-phase and three-phase inverters, and different controls.

The overall efficiency of the grid-connected solar PV system is predicted to be improved in the near future and the cost will be reduced. This analysis study will assist engineers in choosing the most appropriate and suitable control technique and inverter topology suitable to the particular power requirements, position, and capacity for grid connection. This survey is also intended to help researchers, engineers, producers and consumers executing in the field of solar energy to expand the use of solar energy and its integration of grid. Furthermore, it will also allow them to pick the suitable topology for their unique application. 


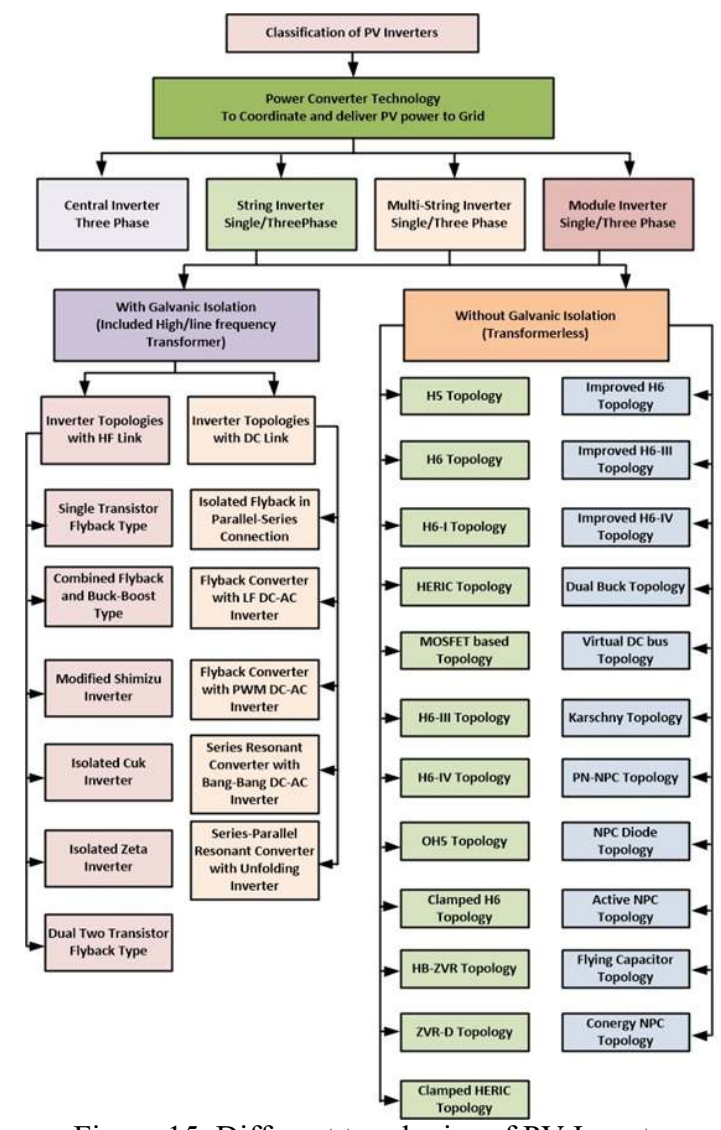

Figure.15. Different topologies of PV Inverter

\section{References}

[1] B. Bijukumar, A. G. K. Raam, S. Ilango Ganesan and C. Nagamani, "Adaptability of grid connected PV inverters with thermoelectric generator as power source: a performance comparison," in IET Power Electronics, vol. 13, no. 5, pp. 981-990, 842020.

[2] A. B. Nassif, "Assessing the Impact of Harmonics and Interharmonics of Top and Mudpump Variable Frequency Drives in Drilling Rigs," in IEEE Transactions on Industry Applications, vol. 55, no. 6, pp. 5574-5583, Nov.-Dec. 2019.

[3] A. Lashab, D. Sera, J. M. Guerrero, L. Mathe and A. Bouzid, "Discrete Model-Predictive-ControlBased Maximum Power Point Tracking for PV Systems: Overview and Evaluation," in IEEE Transactions on Power Electronics, vol. 33, no. 8, pp. 7273-7287, Aug. 2018

[4] P. Siratarnsophon, K. W. Lao, D. Rosewater and S. Santoso, "A Voltage Smoothing Algorithm Using Energy Storage PQ Control in PV-Integrated Power Grid," in IEEE Transactions on Power Delivery, vol. 34, no. 6, pp. 2248-2250, Dec. 2019.

[5] A. Benali, M. Khiat, T. Allaoui and M. Denaï, "Power Quality Improvement and Low Voltage Ride Through Capability in Hybrid Wind-PV Farms Grid-Connected Using Dynamic Voltage Restorer," in IEEE Access, vol. 6, pp. 68634-68648, 2018. 
[6] A. Sangwongwanich, Y. Yang, D. Sera, H. Soltani and F. Blaabjerg, "Analysis and Modeling of Interharmonics From Grid-Connected Photovoltaic Systems," in IEEE Transactions on Power Electronics, vol. 33, no. 10, pp. 8353-8364, Oct. 2018

[7] E. Rikos, S. Tselepis, C. Hoyer-Klick and M. Schroedter-Homscheidt, "Stability and Power Quality Issues in Microgrids Under Weather Disturbances," in IEEE Journal of Selected Topics in Applied Earth Observations and Remote Sensing, vol. 1, no. 3, pp. 170-179, Sept. 2008.

[8] C. Chen, "Virtual Multifunction Power Quality Analyzer Based on Adaptive Linear Neural Network," in IEEE Transactions on Industrial Electronics, vol. 59, no. 8, pp. 3321-3329, Aug. 2012.

[9] L. Asiminoaei, R. Teodorescu, F. Blaabjerg and U. Borup, "Implementation and test of an online embedded grid impedance estimation technique for PV inverters," in IEEE Transactions on Industrial Electronics, vol. 52, no. 4, pp. 1136-1144, Aug. 2005.

[10] A. R. Oliva and J. C. Balda, "A PV dispersed generator: a power quality analysis within the IEEE 519," in IEEE Transactions on Power Delivery, vol. 18, no. 2, pp. 525-530, April 2003.

[11] B. Guha, R. J. Haddad and Y. Kalaani, "Voltage Ripple-Based Passive Islanding Detection Technique for Grid-Connected Photovoltaic Inverters," in IEEE Power and Energy Technology Systems Journal, vol. 3, no. 4, pp. 143-154, Dec. 2016.

[12] M. Tedde and K. Smedley, "Anti-Islanding for Three-Phase One-Cycle Control Grid Tied Inverter," in IEEE Transactions on Power Electronics, vol. 29, no. 7, pp. 3330-3345, July 2014.

[13] L. Dalla Santa, T. Caldognetto, P. Magnone and P. Mattavelli, "Implementation of an active RLC load for unintentional islanding test," 2016 18th European Conference on Power Electronics and Applications (EPE'16 ECCE Europe), Karlsruhe, 2016, pp. 1-8.

[14] General requirements for the competence of testing and calibration laboratories, ISO/IEC Std. 17025:2005, 2010.

[15] Safety of power converters for use in photovoltaic power systems -Part 1: General requirements, IEC Std. 62109-1, April 2010.

[16] Safety of power converters for use in photovoltaic power systems -Part 2: Particular requirements for inverters, IEC Std. 62109-2, June 2011.

[17] Electromagnetic compatibility (EMC) - Part 6-3: Generic standards -Emission standard for residential, commercial and light-industrial environments, IEC Std. 61000-6-3, Feb. 2011.

[18] M. A. Hannan, Z. A. Ghani, A. Mohamed and M. N. Uddin, "Real-Time Testing of a Fuzzy-LogicController-Based Grid-Connected Photovoltaic Inverter System," in IEEE Transactions on Industry Applications, vol. 51, no. 6, pp. 4775-4784, Nov.-Dec. 2015.

[19] S. A. Saleh, C. R. Moloney and M. A. Rahman, "Analysis and Development of Wavelet Modulation for Three-Phase Voltage-Source Inverters," in IEEE Transactions on Industrial Electronics, vol. 58, no. 8, pp. 3330-3348, Aug. 2011.

[20] F. Gao, P. C. Loh, F. Blaabjerg and D. M. Vilathgamuwa, "Performance Evaluation of Three-Level Z-Source Inverters Under Semiconductor-Failure Conditions," in IEEE Transactions on Industry Applications, vol. 45, no. 3, pp. 971-981, May-june 2009.

[21] C. R. Hewson, M. Sumner, G. M. Asher and P. W. Wheeler, "Dynamic mechanical load emulation test facility to evaluate the performance of AC inverters," in Power Engineering Journal, vol. 14, no. 1, pp. 21-28, Feb. 2000.

[22] J. A. Donahue, D. A. Fisher, B. D. Raling and P. J. Tatro, "Performance testing of the Sandy Pond HVDC converter," in IEEE Transactions on Power Delivery, vol. 8, no. 1, pp. 422-428, Jan. 1993.

[23] M. F. Alves and T. N. Ribeiro, "Voltage sag: an overview of IEC and IEEE standards and application criteria," 1999 IEEE Transmission and Distribution Conference (Cat. No. 99CH36333), New Orleans, LA, USA, 1999, pp. 585-589 vol.2.

[24] D. Gallo, C. Landi, M. Luiso and E. Fiorucci, "Survey on Voltage Dip Measurements in Standard Framework," in IEEE Transactions on Instrumentation and Measurement, vol. 63, no. 2, pp. 374387, Feb. 2014

[25] D. Darmawardana et al., "Investigation of high frequency emissions (supraharmonics) from small, grid-tied, photovoltaic inverters of different topologies," 2018 18th International Conference on Harmonics and Quality of Power (ICHQP), Ljubljana, 2018, pp. 1-6. 
[26] A. Hoke, S. Chakraborty and T. Basso, "Testing advanced photovoltaic inverters conforming to IEEE standard 1547 - Amendment 1," 2014 IEEE 40th Photovoltaic Specialist Conference (PVSC), Denver, CO, 2014, pp. 1014-1021.

[27] G. Kim, H. Lee, S. Oh, C. Lee and S. Park, "Study on Real-Time Load Simulator for Testing Propulsion Inverter Test," 2006 SICE-ICASE International Joint Conference, Busan, 2006, pp. 3858-3863.

[28] R. J. Bravo, S. Robles and R. Salas, "Evaluation of German 3-phase solar PV inverter," 2014 IEEE PES General Meeting | Conference \& Exposition, National Harbor, MD, 2014, pp. 1-5.

[29] IEEE Standard for Interconnecting Distributed Resources with Electric Power Systems Amendment 1," in IEEE Std 1547a-2014 (Amendment to IEEE Std 1547-2003), vol., no., pp.1-16, 21 May 2014.

[30] H. Jedtberg, A. Pigazo, M. Liserre and G. Buticchi, "Analysis of the Robustness of Transformerless PV Inverter Topologies to the Choice of Power Devices," in IEEE Transactions on Power Electronics, vol. 32, no. 7, pp. 5248-5257, July 2017, doi: 10.1109/TPEL.2016.2612888.

[31] S. Saridakis, E. Koutroulis and F. Blaabjerg, "Optimal Design of Modern Transformerless PV Inverter Topologies," in IEEE Transactions on Energy Conversion, vol. 28, no. 2, pp. 394-404, June 2013, doi: 10.1109/TEC.2013.2252013.

[32] J. Roy, Y. Xia and R. Ayyanar, "Performance evaluation of single-phase transfomer-less PV inverter topologies," 2018 IEEE Applied Power Electronics Conference and Exposition (APEC), San Antonio, TX, 2018, pp. 3250-3255, doi: 10.1109/APEC.2018.8341568.

[33] K. S. Kumar, A. Kirubakaran and N. Subrahmanyam, "Bi-Directional Clamping Based H5, HERIC and H6-Type Transformerless Inverter Topologies with Improved Modulation Technique," 2020 IEEE International Conference on Power Electronics, Smart Grid and Renewable Energy (PESGRE2020), Cochin, India, 2020, pp. 1-6, doi: 10.1109/PESGRE45664.2020.9070590.

[34] X. Zhang, M. Wang, T. Zhao, W. Mao, Y. Hu and R. Cao, "Topological comparison and analysis of medium-voltage and high-power direct-linked PV inverter," in CES Transactions on Electrical Machines and Systems, vol. 3, no. 4, pp. 327-334, Dec. 2019, doi: 10.30941/CESTEMS.2019.00044.

[35] I. C. Rath and A. Shukla, "Review of Three Phase Transformer-less PV Converters," 2019 IEEE International Conference on Sustainable Energy Technologies and Systems (ICSETS), Bhubaneswar, India, 2019, pp. 063-068, doi: 10.1109/ICSETS.2019.8745120.

[36] K. Zeb et al., "An Overview of Transformerless Inverters for Grid Connected Photovoltaic System," 2018 International Conference on Computing, Electronic and Electrical Engineering (ICE Cube), Quetta, 2018, pp. 1-6, doi: 10.1109/ICECUBE.2018.8610988.

[37] M. Bouzguenda, A. Gastli, A. H. A. Badi and T. Salmi, "Solar photovoltaic inverter requirements for smart grid applications," 2011 IEEE PES Conference on Innovative Smart Grid Technologies Middle East, Jeddah, 2011, pp. 1-5, doi: 10.1109/ISGT-MidEast.2011.6220799.

[38] S. Chaudhary, Z. Ahmad and S. N. Singh, "Single Phase Grid Interactive Solar Photovoltaic Inverters: A Review," 2018 National Power Engineering Conference (NPEC), Madurai, 2018, pp. 1-6, doi: 10.1109/NPEC.2018.8476767.

[39] Ali Khan, M.Y.; Liu, H.; Yang, Z.; Yuan, X. A Comprehensive Review on Grid Connected Photovoltaic Inverters, Their Modulation Techniques, and Control Strategies. Energies 2020, 13, 4185.

[40] Liu, W.; Niazi, K.A.K.; Kerekes, T.; Yang, Y. A Review on Transformerless Step-Up Single-Phase Inverters with Different DC-Link Voltage for Photovoltaic Applications. Energies 2019, 12, 3626.

[41] Lavanya Prabha, S., Gopalakrishnan, M., Neelamegam, M., Development of high-strength nanocementitious composites using copper slag, ACI Materials Journal , 2020, 117(4), pp. 37-46.

[42] M. Tholkapiyan, A.Mohan, Vijayan.D.S , "A survey of recent studieson chlorophyll variation in Indian coastal waters”, IOP Conf. Series: Materials Science and Engineering 993 (2020) 012041, doi:10.1088/1757-899X/993/1/012041. 